\title{
Monolithic Integration of a Nuclear Radiation Sensor and Transistors on High-Purity Silicon
}

\author{
SARAH A. AUDET, MEMBER, IEEE, AND S. E. WOUTERS
}

\begin{abstract}
The physical operation of nuclear radiation sensors is briefly reviewed. This is followed by a description of a typical experimental measurement-system setup, which can be divided into two parts: the application-specific part (including the detector and its associated electronics) and the computer-interfaced part (consisting of a multichannel analyzer). A number of recent improvements in the system design are discussed, and an extended description and a physical example are given of one of the most promising new ideas-the monolithic integration of both the radiation sensor and its associated electronics on one high-ohmic silicon substrate. The results proved to be a stimulus for making nuclear radiation sensors smart enough for direct digital input into computer systems.
\end{abstract}

\section{Radiation Sensor Operation}

$\mathrm{N}$ UCLEAR PARTICLES and high-energy electromagnetic radiation have traditionally been detected with such materials and devices as photographic films, Geiger counters, bubble chambers, and gas-ionization chambers. McKay was the first in 1951 to use a reverse-biased semiconductor (germanium) diode as a method to detect nuclear radiation, thereby beginning the development of semiconductor nuclear radiation sensors [1]. Since that time, other semiconducting materials ( $\mathrm{CdTe}, \mathrm{HgI}_{2}, \mathrm{Ge}(\mathrm{Li}), \mathrm{Si}(\mathrm{Li})$, high-purity $\mathrm{Ge}$, highpurity $\mathrm{Si}$ ) have also been used in the fabrication of nuclear radiation sensors.

The instantaneous response provided by semiconductor detectors gives them a distinctive edge over $\mathrm{X}$-ray films. Their electrical responses can be directly input, after amplification and shaping, into a computer for further processing and storage. In addition, the sensitivities and dynamic ranges of certain semiconductor sensor arrays have been found to be two to three orders of magnitude higher than those values associated with photographic films [2]. Moreover, due to their higher densities and lower ionization energies, semiconductor detectors have better stopping powers and energy resolutions than gaseous detectors.

Although germanium was the first semiconducting material used to detect nuclear radiation, silicon has a larger band gap. Silicon detectors can therefore be operated at room temperature, whereas $\mathrm{Ge}$, high-purity $\mathrm{Ge}, \mathrm{Ge}(\mathrm{Li})$, and $\mathrm{Si}(\mathrm{Li})$ detectors must be cooled to around $77 \mathrm{~K}$ to limit their leakage currents. In addition, silicon radiation sensors can reliably be fabricated by applying the techniques utilized in microelectronic circuit fabrication. (Due to the relatively high densities of CdTe and

Manuscript received June 24, 1989; revised August 28, 1989.

The authors are with the Department of Electrical Engineering, Delft University of Technology, P.O. Box 5031,2600 GA, Delft, The Netherlands. IEEE Log Number 8933032

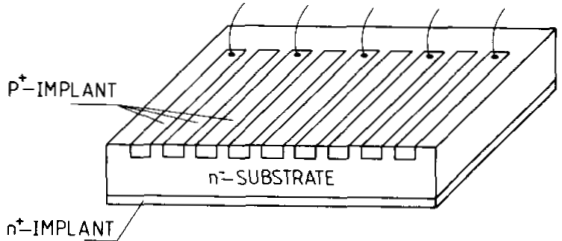

Fig. 1. Schematic of one-dimensional silicon strip detector.

$\mathrm{HgI}_{2}$, these materials are generally used to detect high-energy gamma photons).

When an ionizing particle or soft X-ray traverses through silicon, it losses energy by creating electron-hole pairs. On the average, the particulate radiation will lose $3.6 \mathrm{eV}$ for every electron-hole pair created. The energy of the incident radiation can be measured by collecting this charge. This can best be accomplished through the use of a reverse-biased diode. By making a one-dimensional array of strip diodes (Fig. 1) or a two-dimensional array of square diodes together with a multiplexing readout scheme (Fig. 2), the detector can be made position-sensitive in one or two dimensions, respectively [2].

As only that charge generated in the depletion region of the reverse-biased semiconductor diode can be collected quickly and efficiently, a feasible signal-to-noise ratio can only be obtained through the use of totally depleted detectors. In increasing the reverse-bias on a silicon detector, the associated depletion region will increase. However, the breakdown phenomenon limits the voltage that can be applied and, consequently, the maximum size of the depletion region. To achieve deep depletion depths, high-purity silicon must therefore be utilized [3]. When the resistivity of the starting material is $4 \mathrm{k} \Omega \cdot \mathrm{cm}$ (phosphorous doping concentrations on the order of $10^{12} \mathrm{~cm}^{-3}$ ), a reverse-bias voltage of approximately $100 \mathrm{~V}$ will fully deplete a wafer $300 \mu \mathrm{m}$ thick. In addition, as the amount of charge generated by the incident radiation is limited $(Q=q E / \epsilon$ for electromagnetic radiation, where $Q$ is the amount of charge created, $q$ is the charge of an electron, $E$ is the energy of the incident radiation, and $\epsilon$ is the ionization energy, or approximately $135 \mathrm{C} / \mu \mathrm{m}$ for minimum ionizing particles), low-noise preamplifiers and amplifiers must be employed in association with the detectors.

\section{System SeT-Up}

For different experiments involving different types of radiation, distinct requirements are made on the detector systems. Principally, for the measurement of nuclear-particle 


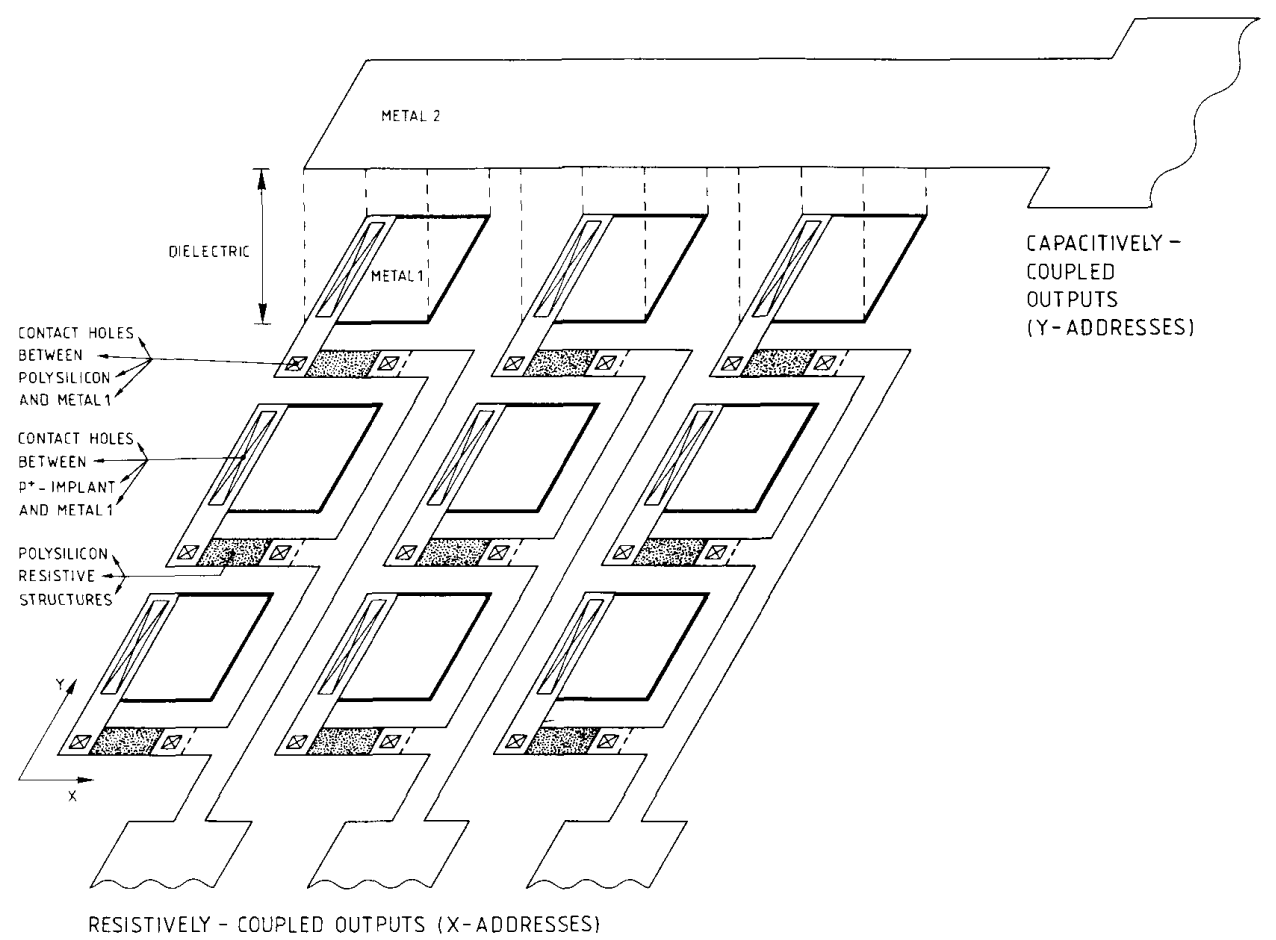

Fig. 2. Schematic of two-dimensional silicon pixel detector [2].

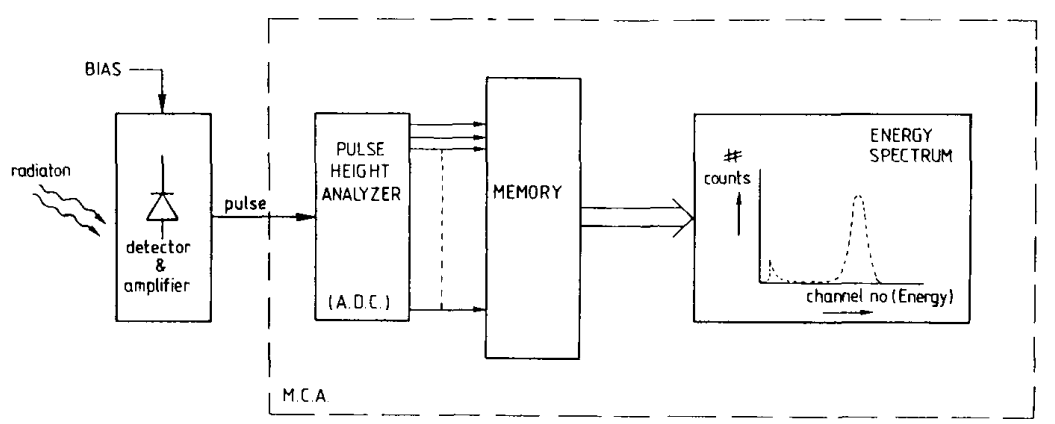

Fig. 3. Measurement system setup for one-element sensor [5].

or soft X-ray radiation, the device must be optimized with respect to energy, space, and time resolutions. These optimization requirements are placed not only on the detector, but also on the front-end electronics, i.e., on the preamplification and amplification units. The measurement system, however, imposes relatively few requirements on the digital-signal processing, which can be performed by means of a computer. The measurement system can therefore be divided into two parts: the application-specific part, i.e., the detector and the detector-related amplification electronics, and the computerinterfaced part, i.e., multichannel analyzers (MCA's). A typical system setup for a one-diode detector is shown in Fig. 3 [3].

\section{Detector-Related Amplification Electronics}

Traditionally, three types of discrete preamplifiers have been utilized: the charge-sensitive, the current-sensitive, and the voltage-sensitive preamplifier. A charge-sensitive preamplifier ideally integrates all the charge collected at that electrode of a radiation sensor with which it is associated onto a feedback capacitor $C_{f}$. Charge-sensitive preamplifiers generally consist of a differential first stage, incorporating a feedback capacitor and often a high-value feedback resistor in parallel. The feedback resistor is a source of noise and, when present, should be made as large as possible so as to minimize its contribution to the preamplifier's total noise. The output voltage $V_{o}$ of the charge-sensitive preamplifier is given by the charge collected by the electrode $Q_{e}$, divided by the feedback capacitance, i.e., $V_{o}=Q_{\mathrm{e}} / C_{f}$. Current-sensitive preamplifiers typically include only a resistor $R_{f}$ in the feedback network. Therefore, the signal from the detector must subsequently be integrated. The integration is performed by the following unit, the spectroscopy amplifier. However, as such, it is designed to convert fast current signals into voltage 
signals. In such a configuration, the voltage output is proportional to the charge collected by the electrode associated with the preamplifier and to the feedback resistor, i.e., $V_{o}(t)$ $=Q_{e} R_{f} \delta(t)$. The output voltage of a voltage-sensitive preamplifier is proportional to the resistive feedback network and inversely proportional to the capacitance of the detector, which is determined by the reverse-bias voltage and can be unstable in time. This instability is minimized when the detector is operated in the fully or overdepleted mode.

The choice regarding which type of preamplifier can optimally be employed depends on the required application, but in all cases, the preamplifier should be one of low noise. The input transistor is primarily responsible for the preamplifier's noise. The input transistor can be fabricated through the use of one of three different existing technologies: bipolar, junction FET (JFET), or MOSFET. The contributions to the overall noise include the thermal noise (predominant in the highfrequency region), the shot noise, and the $1 / f$ noise (predominant in the low-frequency region). Typical values for the equivalent thermal noise voltage per $\mathrm{Hz}^{1 / 2}$ are $1 \mathrm{nV}$ for the bipolar, less than $1 \mathrm{nV}$ for the JFET, and $10 \mathrm{nV}$ for the MOSFET. The shot noise in FET and bipolar transistors is proportional to the gate current and the base current, respectively. It is generally lowest in MOSFET transistors and can be as much as six orders of magnitude higher in bipolar transistors. The $1 / f$ noise is usually negligible in both bipolar and JFET transistors above $1 \mathrm{kHz}$, while it can be of importance in MOSFET's up to 1 MHz. JFET's are most frequently utilized as the input transistors in low-noise preamplifier circuits [4].

\section{Computer Processing}

Due to the extensive research performed in the area of nuclear radiation detectors during the last decade, silicon strip and pixel detectors with thousands of outputs have become available. Each of these strips or pixels must discriminately be read out to obtain position information and each output voltage pulse must also be measured to procure the energy of the incident radiation. In a typical high-energy physics experiment, as many as 10000 outputs are read out during one experiment [5]. A computer system is therefore necessary to handle the signal processing. Large spectroscopy systems for the simultaneous measurement of the energy and the position of radiation also have the need for computer processing of the detector output signals [6]. In the field of medicine, detectors have been employed in the imaging of coronary arteries. The use of a computer for data storage and processing allows the signals obtained from the detectors to be analyzed in the formation of two-dimensional images. However, three-dimensional imaging of the coronary arteries (computer tomography) still remains difficult, due to the beating action of the heart [7].

\section{ImPRovements in System Design}

In the last few years, a shift from pure detector research to research on detector-related electronics has occurred. A number of complications have arisen as a result. First, it is nearly impossible to bond successfully each of the thousands of detector outputs, which can be separated by as little as 10

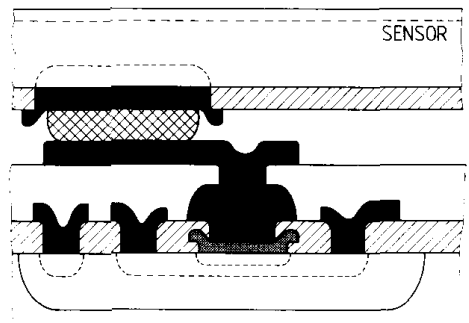

\section{ELECTRONICS}

Fig. 4. "Flip-chip" readout technique. Two separate chips, i.e., detector chip and microelectronics chip, are bonded through use of indium-bump technology [14]

$\mu \mathrm{m}$, involved in some experiments. In addition, a readout system that consists of discrete components is in most cases extensively spacious, as each detector output requires its own preamplifier and amplifier. Also, the longer the interconnections between the detector and the amplifier chain are made, the more signal attenuation and signal disturbance will occur. Finally, due to the increasingly smaller noise associated with recently developed silicon nuclear radiation detectors [8], there has been an increasing need for improvement in the noise performance of the detector-related amplification electronics. These obstacles have led to research in a number of different directions. Attention has turned toward multiplexing and connection techniques, toward the fabrication of specialpurpose low-noise amplification electronics, toward the twodimensional fabrication of detectors and electronics in separate but adjoined materials, and toward the possibility of monolithic readout.

With respect to multiplexing, sample and hold systems have been realized [9], as well as the combination of a silicon strip detector with a charge-coupled device (CCD) multiplexor [10]. In addition, a separate multiplexing scheme has been developed and successfully demonstrated that reduces the number of necessary output connections from $n$, the number of sensing elements, to $2 n^{1 / 2}$ [11].

One interesting connection technique involves the construction of two separate chips, one being the detector and the other the detector-related electronics, with their subsequent bonding through the use of indium-bump technology (Fig. 4) [12]. This "flip-chip" readout method enables the separate optimization of each chip and requires less space, but the technology is difficult and has yet to be perfected.

A recently fabricated 128 -channel NMOS VLSI microplex chip is an example of research being conducted into specialpurpose low-noise amplification electronics [13]. The readout channels have a pitch of $47.5 \mu \mathrm{m}$. Two Microplex chips have been tested with a 256-strip silicon detector with a $25-\mu \mathrm{m}$ strip pitch. A single multiplexed output provides voltages proportional to the integrated charge from each strip.

Other ideas being considered include the fabrication of the detectors in an epilayer of amorphous silicon on top of the detector-related electronics (Fig. 5), or the fabrication of the electronics in an SOI top-layer above the detector (Fig. 6), which would be fabricated in a standard manner in high-ohmic silicon. Both ideas hold promise, as they both allow a 100- 


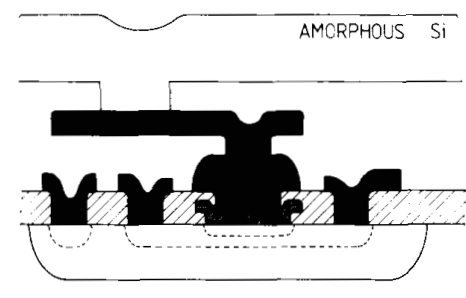

ELEC TRONICS

Fig. 5. Schematic depicting fabrication of radiation detector in epilayer of amorphous silicon on top of detector-related electronics [14].

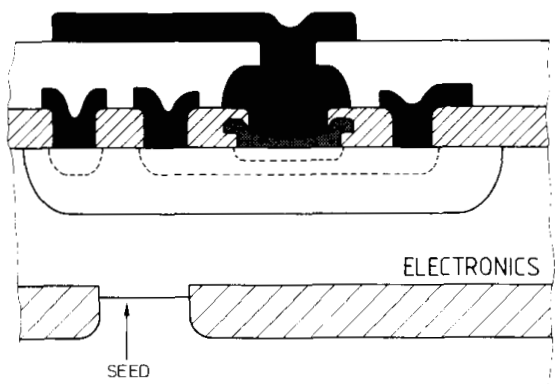

SENSOR

Fig. 6. Schematic depicting fabrication of detector-related electronics in SOI top-layer above detector [14].

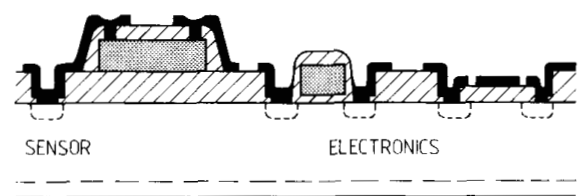

Fig. 7. Monolithic integration of both detector and detector-related electronics on same high-purity silicon substrate.

percent fill factor. However, the detector sensitivity would be limited in the latter design [14].

A number of scientists are presently researching the possibility of fabricating fully integrated detectors in monolithic arrays, where the detector-related amplification electronics would be fabricated on the same high-resistivity silicon as the detector diodes (Fig. 7) [13]-[17]. It would be very favorable to integrate front-end electronics together with radiation detectors onto one chip. Low-noise amplification could theoretically take place, as the high parasitic capacitances associated with off-chip bonding, which cause signal reduction and interference, would be eliminated at this early stage. A reduction in the number of output leads could be envisioned if a multiplexor could be integrated in highresistivity silicon. The total power, size, and wiring costs would also be reduced.

Points to be considered for each different application include the system's spatial and multiple-particle precisions in one and two dimensions, the efficiency of the detector for sensing the designated radiation of interest, the system response time, the percent fill factor, and the radiation hardness of the system. For instance, for application in vertex detection, detectors are needed which have fine spatial and multiple-particle resolutions, have fast response times and that are radiation hard. The same requirements are stated for most experiments with synchrotron radiation, with the addition that a large dynamic range is also necessary.

\section{Processing of Smart High-Purity Silicon Radiation DETECTORS}

The compatible integration of electronics and radiation detectors onto one chip poses many problems. As mentioned earlier, the detector must be fabricated on a high-resistivity substrate $(500-10000 \Omega \cdot \mathrm{cm})$ and it must be biased with high voltages $(30-200 \mathrm{~V})$. The traditional fabrication of electronics, on the other hand, demands a low-resistivity substrate $(<50 \Omega \cdot \mathrm{cm})$ and moderate biasing $(<15 \mathrm{~V})$. In addition, the extra processing steps required for realization of the electronics must not subversively affect the detector operation. Silicon with a (111) orientation is normally used in detector fabrication, as this material is available with a high resistivity. Conversely, MOS circuitry is optimally fabricated on (100) oriented silicon, due to the lower densities of oxide charges and surface states associated with this orientation. However, high-resistivity $(100)$ oriented silicon is beginning to become more widely available.

The incompatibilities existing between the detectors and the electronics can be overcome either by designing special transistors in high-ohmic silicon with the concurrent utilization of isolation techniques or by the creation of a low-ohmic well, in which the electronics will be integrated, within the highohmic silicon. Some ideas and results have been published concerning MOSFET transistors on high-ohmic silicon [13][15], JFET's on high-ohmic silicon [16], and MOSFET transistors in a low-ohmic well within high-resistivity silicon material [17]. A process has also been developed within our laboratory for the fabrication of two-dimensionally position-sensitive radiation sensor arrays and mos-based electronic circuitry on the same high-resistivity silicon substrate. The process is based on the use of ion-implantation for the fabrication of the source, drain and detector junctions and on the use of doublelayer metalization for realizing the passive multiplexing readout scheme associated with the sensor array. This process has been thoroughly described [18], [19]. Detector prototypes and test structures, consisting of a nuclear radiation imaging sensor with 256 array elements, $250 \times 250 \mu \mathrm{m}^{2}$ in size [19], various size diodes, and mos capacitors, were fabricated together with numerous $p$-channel (polysilicon and metal gate) MOS transistors of different widths and lengths using the process described in [18]. A photograph of the sensor array with various test structures, fabricated up to the first layer metalization, is shown in Fig. 8(a), while Fig. 8(b) shows a close-up of the sensor array and the mos transistors.

Measurements demonstrated that the detector diodes were of good quality [19]. Fig. 9 depicts the leakage current measurement of one of the resistive outputs of the sensor array, i.e., 16 sensing elements in parallel, after the first level metalization (I1) and upon full fabrication (I2). The transistors demonstrated characteristic behavior. Fig. 10 shows the dc 


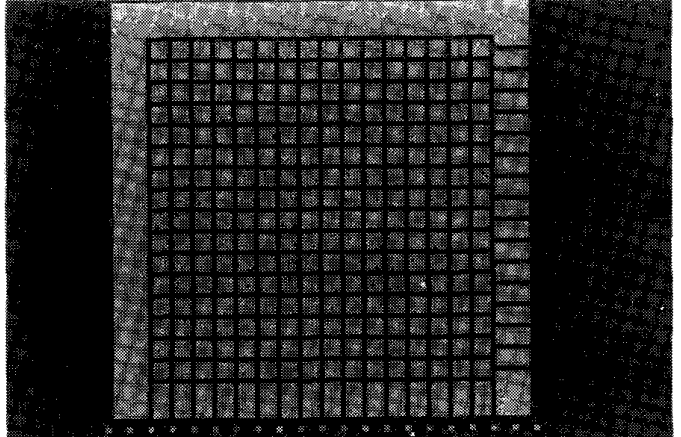

(a)

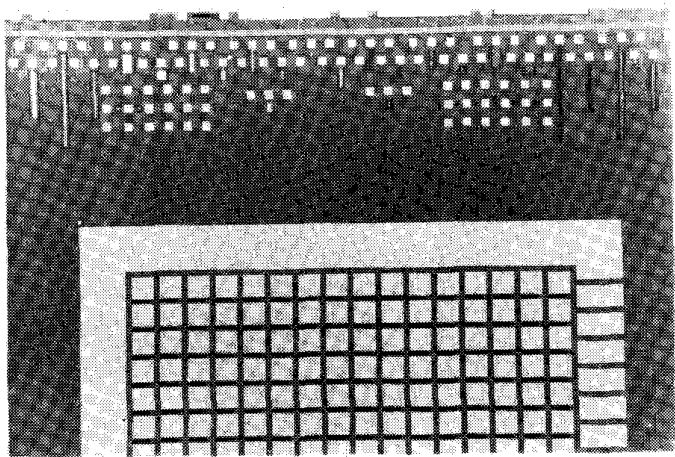

(b)

Fig. 8. (a) Photograph of 256 element nuclear radiation sensor, fabricated up to first level metalization. (b) Close-up photograph of sensor with polysilicon and metal gate MOS transistors.

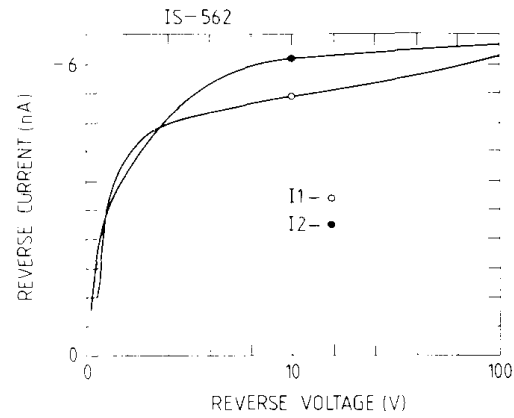

Fig. 9. Typical reverse $I-V$ curve obtained from a resistive output of sensor array, i.e. 16 sensor elements in parallel, at room temperature after the first level metalization (I1) and after full fabrication (I2) [19].

characteristics of a typical transistor. No punchthrough was observed below $15 \mathrm{~V}$, while the threshold voltage $V_{t}$ was found to vary between 1 and $-3 \mathrm{~V}$. At the drain-source bias current of $200 \mu \mathrm{A}$, the small-signal transconductance was $45 \mu \mathrm{A} / \mathrm{V}$.

\section{CONCLUSION}

After many years of intense research into high-purity silicon radiation sensors all over the world, much knowledge has been gained concerning the fabrication and the operation of radiation detectors. Limitations now exists in the detectorrelated electronics part of the sensor system. Several possible solutions have been briefly described. One of these solutions, i.e., the monolithic integration of transistors and detectors

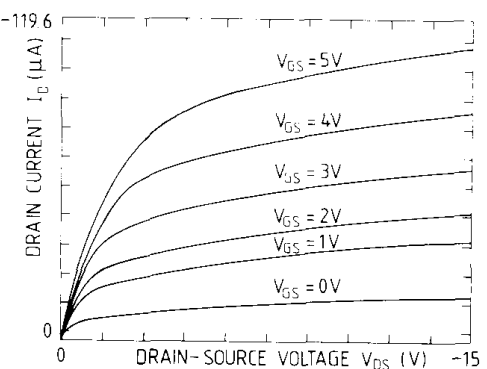

Fig. 10. DC characteristics of typical polysilicon gate MOS transistor of width $30 \mu \mathrm{m}$ and length $14 \mu \mathrm{m}$. $V_{\mathrm{OS}}$ varies from $0-5 \mathrm{~V}$.

onto one high-ohmic silicon substrate, has been further investigated in our laboratory. Only a few advances have yet been made in the field of integrating front-end electronics on the detector chip. This paper presents a number of solutions to the incompatibility problems existing for the co-integration of the detector and the electronics. Through the use of a specialized processing sequence [18], radiation sensors and mos-based electronic circuitry have been fabricated on the same high-resistivity silicon substrate with the concurrent maintanence of good detector characteristics. The results are thus a stimulus for making radiation sensors smart enough for direct digital input into computer systems.

\section{ACKNOWLEDGMENT}

The authors are indebted to Dr. A. Schmitz of Philips Research Laboratories in Eindhoven, The Netherlands, for the advice and consultation received during our many profitable discussions with him. We would also like to thank the members of the I.C. Laboratory at Delft University of Technology and Mssrs. H. Maas and J. Cox of Philips Research Laboratories in Eindhoven, for helping in the fabrication of the device.

\section{REFERENCES}

[1] K. McKay, "Electron-hole production in germanium by alpha particles," Phys. Rev., vol. 84, pp. 829-832, 1951

[2] S. A. Audet, "Radiation sensor array fabricated on high-purity silicon with coupling impedances realized through polysilicon and doublelayer metalization layers." in Proc. 1988 Electronic Devices and Materials Symp., Kaohsiung, Taiwan, R.O.C., Aug. 19-21, 1988, pp. 212-217.

[3] W. R. Th. ten Kate, "Detectors for nuclear radiation," Sensors and Actuators, vol. 10, pp. 83-101, 1986.

[4] E. H. M. Heijne and P. Jarron, "The impact of microelectronics on particle detection," Nucl. Instrum. Methods, vol. 226, pp. 12-15, 1984

[5] W. R. Th. ten Kate, Ph.D. dissertation, Delft Univ. of Technology, Delft, The Netherlands, 1987.

[6] R. Nessel, N. Pilz, P. Hoffman, and K. H. Lieser, "X-ray and gammaray spectrometer based on a 68000 microprocessor computer for simultaneous measurement and evaluation," Nucl. Instrum. Methods, vol. A267, pp. 157-160, 1988

[7] A. C. Thompson, R. Hofstadter, J. N. Otis, H. D. Zeman, R. S Kernoff, E. Rubenstein, J. C. Giacomini, H. J. Gordon, G. S. Brown. and W. Thomlinson, "Transvenous coronary angiography using synchrotron radiation." Nucl. Instrum. Methods, vol. A266, pp. $252-259.1988$

[8] J. Kemmer and G. Lutz, "New detector concepts," Nucl. Instrum. Methods, vol. A253, pp. 365-377, 1987.

[9] J. T. Walker, S. Parker, B. Hymans, and S. L. Shapiro, "Development of high density readout for silicon strip detectors," Nucl. Instrum. Methods, vol. 226, pp. 200-203, 1984 
[10] W. R. Th. ten Kate and C. L. M. van der Klauw, "Strip-detector-CCD coupling by means of a bipolar transistors," Nucl. Instrum. Methods, vol. 226, pp. 193-195, 1984.

[11] S. A. Audet and E. M. Schooneveld, "High-purity silicon radiation sensor array," Nucl. Instrum. Methods., vol. A275, pp. 517-526, 1989.

[12] S. L. Shapiro, W. M. Dunwoodie, J. F. Arens, J. G. Jernigan, and S. Gaalema, "Silicon PIN diode array hybrids for charged particle detection," Nucl. Instrum. Methods, vol. A275, pp. 580-586, 1989.

[13] G. Anzivino, R. Horisberger, L. Hubbeling, B. Hyams, S. Parker, A. Breakstone, A. M. Litke, J. T. Walker, and N. Blingefors, "First results from a silicon strip detector with VLSI readout," Nucl. Instrum. Methods, vol. A243, pp. 153-158, 1986.

[14] B. Dierickx, "XYW detector: A smart two-dimensional particle sensor," Nucl. Instrum. Methods, vol. A275, pp. 542-544, 1989.
[15] S. Holland, "An IC-compatible detector process," presented at the IEEE Nuclear Science Symp., Orlando, FL., Nov. 9-11, 1988.

[16] V. Radeka, P. Rehak, S. Rescia, E. Gatti, A. Longoni, M. Sampietro, G. Bertuccio, P. Holl, L. Struder, and J. Kemmer, "JFET for completely depleted high-resistivity silicon," presented at ESSDERC, Montpellier, France, Sept. 13-16, 1988.

[17] G. Vanstraelen, I. Debusschere, C. Claeys, and G. Declerck, "Fully integrated CMOS pixel detector for high energy particles," Nucl. Instrum. Methods, vol. A275, pp. 574-579, 1989

[18] S. E. Wouters, S. A. Audet, and M. H. Kim, "Compatible integration of radiation detectors and transistors," in Proc. Transducers '89, Montreux, Switzerland, June 25-30, 1989, p. 178.

[19] S. A. Audet, E. M. Schooneveld, S. E. Wouters, and M. H. Kim, "High-purity silicon soft X-ray imaging sensor array," in Proc. Transducers '89, Montreux, Switzerland, June 25-30, 1989, p. 172. 